\title{
Investigation of heat transfer in the cup-cast method by experiment, and analytical method
}

\author{
F. Pahlevani, J. Yaokawa \& K. Anzai \\ Department of Metallurgy, School of Engineering, Tohoku University, \\ Sendai, Japan
}

\begin{abstract}
Semi-solid metal alloys have a special microstructure of globular grains suspended in a liquid metal matrix that cause a significant number of benefits. A new semi-solid casting method (cup-cast method) that has been recently developed has eliminated all of the difficulties associated with other semi-solid casting processes and makes semi-solid casting as easy as pouring water from a pitcher into a drinking glass. This method is based on the nucleation and growth of solid-particles. Heat transfer phenomenon is one of the most important factors in this method that governs the shape, size, and fraction of solid particles. In this study, the heat transfer phenomena in the cup cast method has been studied experimentally and theoretically. First, the temperature distribution was measured at a semi-solid casting of Al-A356 with the cup-cast method at different points. Finally, a model for heat transfer phenomena was proposed. The final model was in good agreement with the experimental data.
\end{abstract}

Keywords: semi-solid, cup-cast, heat transfer, analytical equation, modelling.

\section{Introduction}

The semi-solid processes of Al-A356 alloy produced globular instead of dendritic alpha phase and extraordinarily fine fibrous aluminum-silicon eutectic structure, which apparently gives exceptionally high ductility [1-4].

Semi-solid processing offers several significant process and product cost advantages, for example: 1- reduced cost of porosity-related scrap, rework and impregnation of die cast type products; 2- reduced material content of squeeze, low pressure and permanent mold type products by casting to near-to-net-shape and by casting thinner sections of high integrity material; 3- long tool life, the 
result of substantially less heat of fusion released to the die and a reduced $\Delta t$ between tool and casting, both delaying the onset of heat checking and other thermal stress induced tool failures [1-4].

Conventional semi-solid casting processes face many potential problems but the cup-cast method that has been recently developed is a novel process that makes semi-solid casting as easy as pouring water from a pitcher into drinking glass, and avoids all of the problems and difficulties associated with other semi-solid casting processes. In this method, on pouring, nucleation may take place both on the wall and throughout the zone that is super cooled by contact with the cold mould. It is likely that this zone would have a thickness of the order of millimeter for a pouring temperature close to the melting point and thinner for a higher temperature. All nuclei that survive at this time are able to grow; they will "drift" away from the interface by convection motion of the liquid and then moved to the other part of the cup that contains low temperature and composition gradient melt.

Modelling and simulation are an important part of process development, providing insight into the mechanisms controlling process performance. On the other hand, one of the specialties of this method (cup-cast method) is its isothermal holding stage that is caused by the heat transfer phenomenon. The aim of this paper is to describe the modelling of the heat transfer phenomenon of the cup cast method and comparing this with the experimental result that shows good agreement between analytical equation of heat transfer and experiment after saturation (isothermal holding).

\section{Temperature measurement}

The Al-A356 alloy with chemical composition as shown in table 1 was melted at the electrical resistance furnace in the graphite crucible at $720^{\circ} \mathrm{C}$ and then under certain conditions poured into the cup (shown schematically in Fig. 1). The temperature was measured at the center of the cup, inside and outside of the cup's wall by $0.1 \mathrm{~mm}$ thickness positive Chromel wire and negative Alumel wire thermocouple. The temperature was recorded during pouring of the melt to the cup and rest time, 20 per second to exact temperature profile could be achieved.

Table 1: Chemical composition of used Al-A356 alloy.

\begin{tabular}{|c|c|c|c|c|c|c|c|}
\hline $\mathrm{Si}$ & $\mathrm{Mg}$ & $\mathrm{Fe}$ & $\mathrm{Cu}$ & $\mathrm{Zn}$ & $\mathrm{Mn}$ & $\mathrm{Sn}$ & $\mathrm{Ni}$ \\
\hline 7.12 & 0.45 & 0.11 & 0.007 & 0.007 & 0.006 & 0.006 & 0.003 \\
\hline
\end{tabular}

At the center of the cup after the pouring start, because it is located at the melt's stream, the temperature climbed to its optimum temperature rapidly. At the inside of the cup's wall the temperature increase was delayed, but reached the melt's flow during turbulence to the point where it increasede up to the same temperature as the center of the cup (there is just a small difference between the temperature of the melt at the center of the cup and inside of the cup's wall so it shows uniform temperature distribution in the cup). By transferring the heat from 
the melt to cup, the temperature of the melt will decrease gradually but uniformly at the semi-solid state (between liquidus " $620{ }^{\circ} \mathrm{C}$ " and Solidus "575 ${ }^{\circ} \mathrm{C}$ " line) and the temperature of the cup will rise up to reach to its saturation point that will be followed by a constant temperature at the cup's wall.

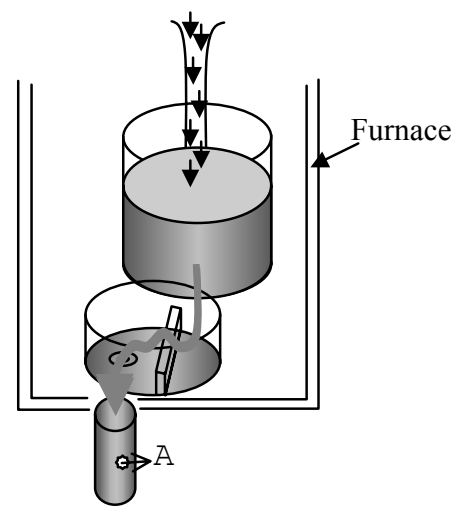

Figure 1: $\quad$ Schematic figure of experimental cup-casting method.

From these curves (Fig. 2(a)) it could be said that after pouring, the heat of the melt transfered only to the cup. Then, by heat transfer the temperature of the melt and temperature of the cup approached a saturation temperature and after some time the cup and melt will keep this saturation temperature and after rest time, decrease gradually. From Fig 2(b) that shows temperature deference at the different height of the cup it could be assumed there is one dimensional heat transfer through melt to the cup.
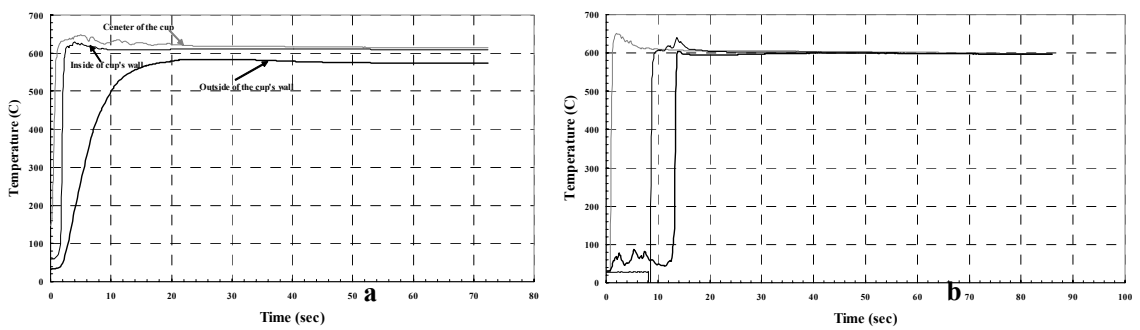

Figure 2: (a) Temperature profile at different point of the cup during CupCast method. (b) Temperature deference in the melt at different height of cup during Cup-Cast method.

\section{Analytical model for heat transfer}

Based on temperature measurement and some assumption (such as constant material properties, one dimensional heat transfer and no-superheating) an analytical model was proposed for heat transfer in cup cast method. 
Fig 3 shows the way of heat transfer through the melt to the air schematically, as it was mentioned temperature was measured at three point (center of the cup, inside and outside of the cup's wall) so analytical model also was done for these three points.

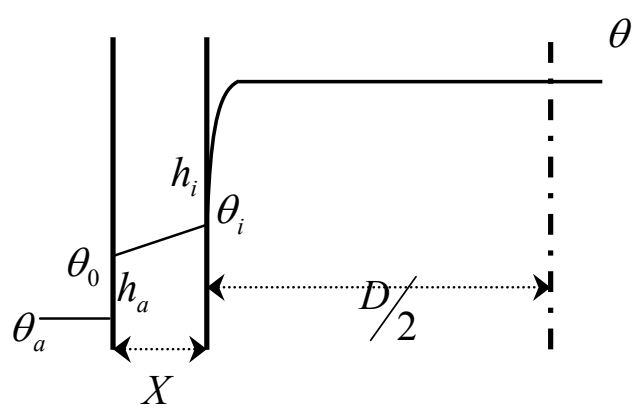

Figure 3: $\quad$ Schematic of heat transfer in cup-cast method.

\subsection{Center of the cup, and inside of the cup's wall}

From heat conservative low and heat balance following equation could be written

$$
\begin{gathered}
h_{a}\left(\theta_{0}-\theta_{a}\right)=\frac{\lambda\left(\theta_{i}-\theta_{0}\right)}{X} \\
\frac{\lambda\left(\theta_{i}-\theta_{0}\right)}{X}=h_{i}\left(\theta-\theta_{i}\right) \\
h_{i}\left(\theta-\theta_{i}\right) \pi D=-\frac{\partial \theta}{\partial t}\left(\rho c+\frac{\rho H_{f}}{\theta_{L}-\theta_{S}}\right) \pi\left(\frac{D}{2}\right)^{2}
\end{gathered}
$$

If $\theta_{0}$ was calculated from eqn (1-a) and introduce to (1-b) eqn (3) could be obtained

$$
\begin{gathered}
\theta_{i}=\frac{h_{i} \theta+\frac{h_{a}(\lambda / X)}{h_{a}+(\lambda / X)} \theta_{a}}{h_{i}+\frac{\lambda}{X}-\frac{(\lambda / X)^{2}}{h_{a}+(\lambda / X)}} \\
\theta-\theta_{i}=\frac{\frac{h_{a} X}{\lambda}}{\left(1+h_{a} X / \lambda\right)\left(1+h_{i} X / \lambda\right)-1}\left(\theta-\theta_{a}\right)
\end{gathered}
$$


By substitution the definition of $\theta-\theta_{i}$ from eqn (3) into eqn (2) and introducing $\beta$ (eqn 4) temperature profile at the center of the cup as function of time will be determine as equation 5

$$
\begin{aligned}
& \beta=\frac{h_{i} 4 / D^{\left(h_{a} x / \lambda\right)}}{\left(\rho c+\frac{\rho H_{f}}{\theta_{L}-\theta_{s}}\right)\left\{\left(1+h_{a} x / \lambda\right)\left(1+h_{i} x / \lambda\right)-1\right\}} \\
& \beta d t=-\frac{1}{\theta-\theta_{a}} d \theta \Rightarrow \theta=\theta_{a}+\left(\theta_{L}-\theta_{a}\right) e^{-\beta t}
\end{aligned}
$$

Thus, eqn (3-a) together with eqn (5) yields the temperature distribution at inside of cup's wall

$$
\theta_{i}=\theta_{a}+\frac{\left(1+\frac{h_{a} X}{\lambda}\right) \frac{X h_{i}}{\lambda}}{\left(1+\frac{X h_{i}}{\lambda}\right)\left(1+\frac{X h_{a}}{\lambda}\right)-1}\left(\theta_{L}-\theta_{a}\right) e^{-\beta t}
$$

\subsection{Outside of cup's wall}

For the temperature profile of the outside of cup's wall, because at the early stage here is not a uniform temperature distribution in the cup, the temperature profile for the first stage (before uniform temperature distribution) is in the form of eqn (7) (that is for semi-infinite solid was located in a uniform temperature media but suddenly on surface of it will heat up by a high temperature fluid), and after that condition it will given by eqn (8)

$$
\theta^{+}=\operatorname{erfc} \frac{x^{+}}{2 \sqrt{t^{+}}}-\exp \left(x^{+}+t^{+}\right) \operatorname{erfc}\left(\frac{x^{+}}{2 \sqrt{t^{+}}}+\sqrt{t^{+}}\right)
$$

where $\theta^{+}=\frac{\theta_{0}-\theta_{a}}{\theta_{L}-\theta_{a}}, x^{+}=\frac{x}{\lambda / h}$, and $t^{+}=\frac{a t}{(\lambda / h)^{2}}$

$$
\theta_{0}=\theta_{a}+\frac{X h_{i} / \lambda}{\left(1+X h_{i} / \lambda\right)\left(1+X h_{a} / \lambda\right)-1}\left(\theta_{L}-\theta_{a}\right) e^{-\beta t}
$$


As it was mentioned, this equation is for semi-infinite solid and because the cup is not as thick as could be considered the same as semi-infinite solid, this equation could not be a suitable analytical solution for this problem, so for finding a more realistic solution a slab $0 \leq x \leq L$ would be considered by mathematical formulation of the problem the same as:

$$
\begin{gathered}
\frac{\partial^{2} \theta}{\partial x^{2}}=\frac{1}{\alpha} \frac{\partial \theta}{\partial t} \text { in } 0<x<L, t>0 \\
\theta(x, t)=\theta_{a} \text { at } x=0, t>0 \\
\frac{\partial \theta}{\partial x}=0 \text { at } x=L, t>0 \\
\theta(x, t)=\theta_{L} \text { for } t=0 \text { in } 0 \leq x \leq L
\end{gathered}
$$

And solve this problem by using the integral method. For the case $\delta(t)<L$ (the thermal layer thickness is less than the slab thickness) the eqn (9-a) was integrated over the thermal layer thickness and obtain

$$
\begin{gathered}
-\left.\alpha \frac{\partial \theta_{0}}{\partial x}\right|_{x=0}=\frac{d}{d t}\left(\gamma-\theta_{a} \delta\right) \\
\text { where } \gamma \equiv \int_{x=0}^{\delta} \theta_{0}(x, t) d x
\end{gathered}
$$

A cubic profile for the temperature as given by eqn (11) was chosen and by applying the conditions given by eqn (12) the coefficients were determined and utilize eqn $(10-b)$ to determine the thermal layer thickness.

$$
\begin{gathered}
\left\{\theta_{0}(x, t)=a+b x+c x^{2}+d x^{3}\right\} \\
\left.\theta\right|_{x=0}=\theta_{a},\left.\theta\right|_{x=\delta}=\theta_{L},\left.\frac{\partial \theta}{\partial x}\right|_{x=\delta}=0,\left.\frac{\partial^{2} T}{\partial x^{2}}\right|_{x=0}=0
\end{gathered}
$$

The resulting temperature profile becomes

$$
\begin{gathered}
\frac{\theta_{0}(x, t)-\theta_{a}}{\theta_{L}-\theta_{a}}=1-3 / 2\left(\frac{x}{\delta}\right)+1 / 2\left(\frac{x}{\delta}\right)^{3} \\
\text { where } \delta=\sqrt{8 \alpha t}
\end{gathered}
$$

This solution is valid for $0 \leq x \leq L$ as long as $\delta \leq L$ and after that eqn (8) shows the temperature profile. 


\section{Comparing the experiment and analytical temperature profile}

Fig 4 and 5 shows the difference between the temperature profile from the analytical equation and the experiment. From Fig. 4 it could be summarized that there is good agreement between the experiment and analytical equation for temperature distribution at the center of cup and inside of cup's wall.

\section{Center of cup}

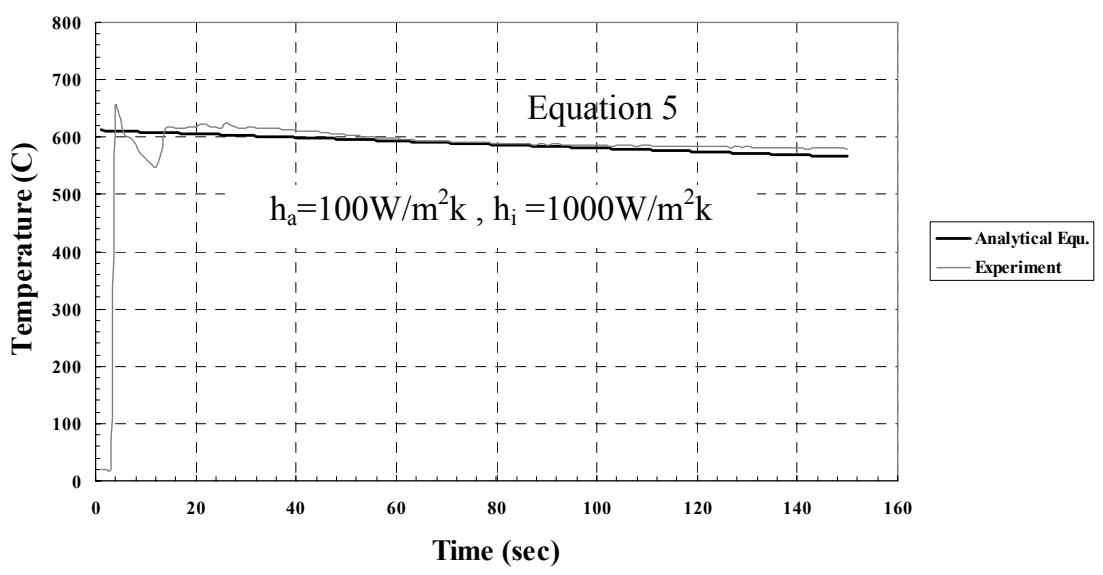

(a)

Inside of cup's wall

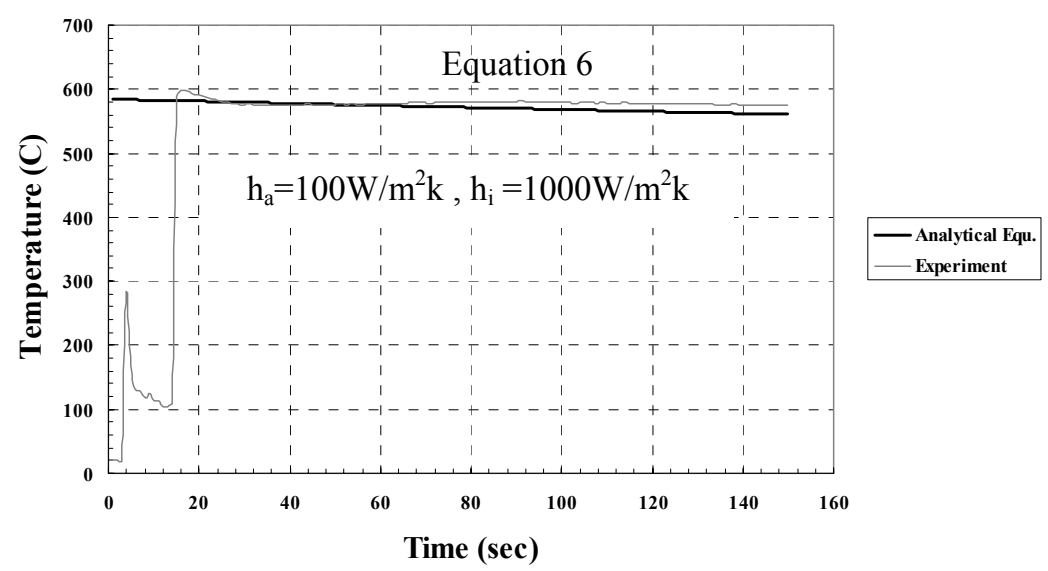

(b)

Figure 4: Comparing of analytical temperature profile and experiment. 


\section{Outside of cup's wall}

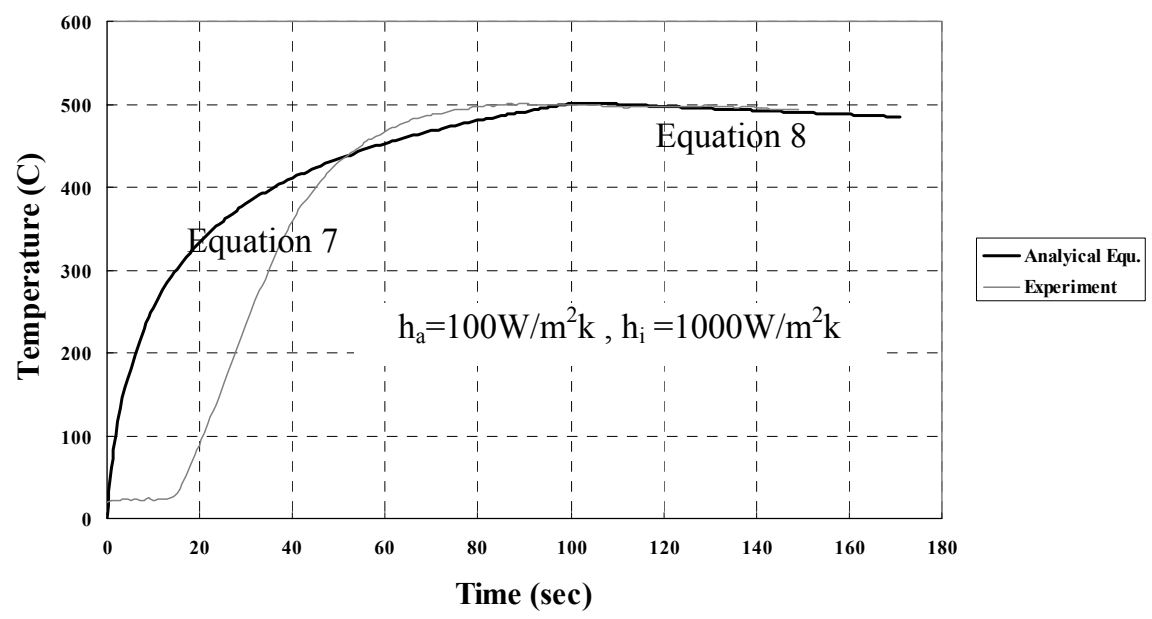

(a)

\section{Outside of Cup's wall}

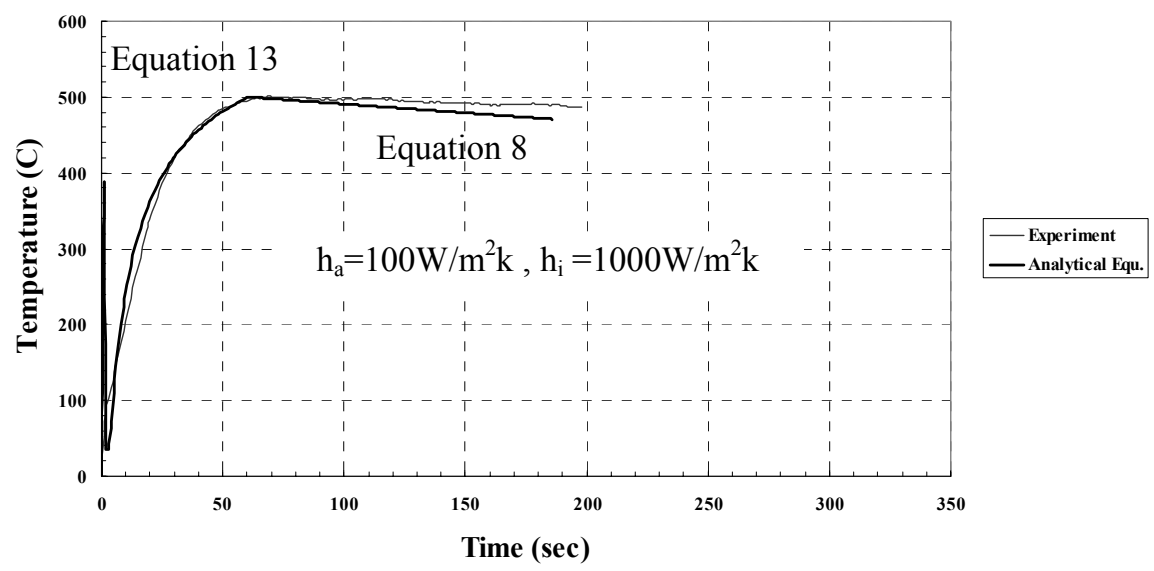

(b)

Figure 5: Comparing of analytical temperature profile and experiment.

Fig. 5(a) shows a combination of equation (8) and (9) could not be a good solution for this problem, but equation (13) for early stage and equation (9) after saturation time (uniform temperature distribution) are in good agreement with experimental result (Fig 5(b)). 


\section{Conclusion}

Cup-cast method is the most convenient method for semi-solid casting that has just been developed by the author. This method is based on the nucleation and growth of solid particles, and sufficient rest time (holding the isothermally in semi-solid state), heat transfer phenomena will considerably influence on the semi-solid microstructure of this method. Based on the facts of this method those driven out from the temperature measurement a model for heat transfer in cup cast method was proposed. This analytical equation is in good agreement with experiments at the center and inside of the cup's wall and is in acceptable agreement with experiment at the outside of the cup's wall.

\section{Index A}

Table 2: $\quad$ List of symbols and their definition in analytical model.

\begin{tabular}{|c|c|c|c|}
\hline$\theta$ & Temperature of the melt & $\theta_{a}$ & Room temperature \\
\hline$D$ & Diameter of cup & $\theta_{0}$ & $\begin{array}{c}\text { Temperature of the cup } \\
\text { outside }\end{array}$ \\
\hline$X$ & Thickness of the cup & $\theta_{i}$ & Temperature of cup inside \\
\hline$h$ & Heat transfer coefficient & $\delta$ & Thermal layer thickness \\
\hline$\lambda$ & $\begin{array}{l}\text { Thermal conductivity of } \\
\text { cup }\end{array}$ & subscript & \\
\hline$\rho$ & Density of cup & $i$ & Between melt and cup \\
\hline$c$ & $\begin{array}{l}\text { Specific heat coefficient } \\
\text { of cup }\end{array}$ & $a$ & Between cup and air \\
\hline$a$ & $\begin{array}{c}\text { Thermal diffusivity of } \\
\text { cup }\end{array}$ & & \\
\hline$L$ & Slab thickness & & \\
\hline
\end{tabular}

\section{References}

[1] J. L. Jorstad, Q. Y. Pan, D. A. Pelian, "Solidification microstructure affecting ductility in semi-solid-cast products", Materials Science and Engineering A, 413-414 (2005) pp. 189-191.

[2] D. Brabazon, D. J. Browne, A. J. Carr, "Experimental investigation of the transient and steady state rheological behavior of Al-Si alloys in the mushy state", Materials Science and Engineering A 356 (2003) pp. 69-80. 
[3] B. P. Gautham, P. C. Kapur, "Rheological model for short duration response of semi-solid metals", Materials Science and Engineering A 393 (2005) pp. 223-228.

[4] J. L. Wang, Y. H. Su, C-Y. A. Tsao, "Structural evolution of conventional cast dendritic and spray-cast non-dendritic structure during isothermal holding in the semi-solid state", Scripta Materialia (USA), No. 12, 37 (1997), pp. 2003-2007. 